PROCEEDINGS OF THE

AMERICAN MATHEMATICAL SOCIETY

Volume 132, Number 4, Pages 1195-1199

S 0002-9939(03)07289-7

Article electronically published on October 15, 2003

\title{
A UNIFORM FOURIER RESTRICTION THEOREM FOR SURFACES IN $\mathbb{R}^{3}$
}

\author{
DANIEL M. OBERLIN
}

(Communicated by Andreas Seeger)

\begin{abstract}
We establish a uniform Fourier restriction estimate for certain hypersurfaces in $\mathbb{R}^{3}$.
\end{abstract}

Suppose $S$ is a smooth hypersurface in $\mathbb{R}^{n}$. Let $\sigma$ be a measure on $S$ given by $d \sigma=\psi d m$ where $m$ is Lebesgue measure on $S$ and $\psi$ is a smooth cutoff function. An argument due to Tomas and Stein (see, e.g., [5]) shows that if $\sigma$ satisfies the Fourier transform estimate

$$
|d \sigma(\xi)| \leq c|\xi|^{-(n-1) / 2}
$$

(which will hold if the Gaussian curvature of $S$ does not vanish on the support of $\psi)$, then there is the Fourier restriction estimate

$$
\left(\int_{S}|\widehat{f}(\xi)|^{2} d \sigma(\xi)\right)^{1 / 2} \leq C(c)\|f\|_{2(n+1) /(n+3)}
$$

for suitable functions $f$ on $\mathbb{R}^{n}$. There are analogues of this result under less stringent curvature conditions (see, e.g., 4]) and even for flat curves and surfaces (see [1] for one of the first of these). The purpose of this note is to prove a version of this Stein-Tomas Fourier restriction theorem for a class of surfaces in $\mathbb{R}^{3}$. Suppose $\gamma$ is a nonnegative $C^{(3)}$ function on $[0, b)$ satisfying $\gamma(0)=\gamma^{\prime}(0)=0, \gamma^{(j)}(t)>0$ for $t>0$ and $j=1,2,3$. Define

$$
\Gamma:\left\{x \in \mathbb{R}^{2}:|x|<b\right\} \rightarrow \mathbb{R}^{3}
$$

by

$$
\Gamma(x)=(x, \gamma(|x|)) .
$$

Let the surface $S$ in $\mathbb{R}^{3}$ be the range of $\Gamma$, and let $\sigma$ be the image under $\Gamma$ of the measure

$$
\left(\frac{\gamma^{\prime}(|x|)}{|x|}\right)^{1 / 2} d x
$$

Theorem 1. There is an absolute constant $C$ such that the inequality

$$
\left(\int_{S}|\widehat{f}(\xi)|^{2} d \sigma(\xi)\right)^{1 / 2} \leq C\|f\|_{4 / 3}
$$

holds for measurable $f$ on $\mathbb{R}^{3}$.

Received by the editors December 30, 2002.

2000 Mathematics Subject Classification. Primary 42B10.

Key words and phrases. Fourier restriction.

The author was partially supported by the NSF. 
Carbery and Ziesler ([2]) raise the possibility of a universal restriction theorem for convex hypersurfaces in $\mathbb{R}^{n}$. They point out that, in the radial case we consider and when $n=3$, the natural choice for a weight is

$$
\left(\frac{\gamma^{\prime \prime}(|x|) \gamma^{\prime}(|x|)}{|x|}\right)^{1 / 4}
$$

the $1 / 4$ power of the affine curvature on $S$. Our choice

$$
\left(\frac{\gamma^{\prime}(|x|)}{|x|}\right)^{1 / 2}
$$

is (unfortunately) smaller since $\gamma^{\prime}(t) \leq t \gamma^{\prime \prime}(t)$. For example, if $\gamma(t)=t^{m}$ for $m>2$, our weight is $m^{1 / 2}|x|^{(m-2) / 2}$ while the $1 / 4$ power of the affine curvature is $\left(m^{3}-m\right)^{1 / 4}|x|^{(m-2) / 2}$.

We regard the uniformity implied by the fact that $C$ is an absolute constant as being the principal interest of the present result. But it is perhaps worth pointing out that our theorem applies to certain flat functions for which the more usual methods, based on Fourier transform estimates, are difficult. An example is given by the choice $\gamma(t)=e^{-1 / t}$ (which satisfies our monotonicity hypotheses on some interval $(0, \delta))$.

Proof of Theorem. By duality it is enough to prove the inequality

$$
\|\widehat{g d \sigma}\|_{4} \leq C\|g\|_{L^{2}(d \sigma)}
$$

for functions $g$ on $S$. By Plancherel's theorem, this is equivalent to

$$
\|(g d \sigma) *(g d \sigma)\|_{2} \leq C\|g\|_{L^{2}(d \sigma)}^{2}
$$

and so to

$\iint g(x) g(y) h(x+y, \gamma(|x|)+\gamma(|y|))\left(\frac{\gamma^{\prime}(|x|) \gamma^{\prime}(|y|)}{|x||y|}\right)^{1 / 2} d x d y \leq C\|g\|_{L^{2}(d \sigma)}^{2}\|h\|_{2}$,

where the integrals are over $\left\{x \in \mathbb{R}^{2}:|x|<b\right\}$. Applying the Schwarz inequality shows that it is enough to establish the inequality

$$
\iint h^{2}(x+y, \gamma(|x|)+\gamma(|y|))\left(\frac{\gamma^{\prime}(|x|) \gamma^{\prime}(|y|)}{|x||y|}\right)^{1 / 2} d x d y \leq C\|h\|_{2}^{2} .
$$

Writing $x=r e^{i \theta}$ and $y=s e^{i \phi}$, the left-hand side of (1) is

$$
\int \cdots \int h^{2}\left(r e^{i \theta}+s e^{i \phi}, \gamma(r)+\gamma(s)\right)\left(r \gamma^{\prime}(r) s \gamma^{\prime}(s)\right)^{1 / 2} d \theta d \phi d r d s .
$$

A Jacobian computation shows that

$$
\begin{aligned}
\iint h^{2} & \left(r e^{i \theta}+s e^{i \phi}, \gamma(r)+\gamma(s)\right) d \theta d \phi \\
& =4 \int_{\{|r-s|<|x|<r+s\}} \frac{h^{2}(x, \gamma(r)+\gamma(s))}{\left(\left[(r+s)^{2}-|x|^{2}\right]\left[|x|^{2}-(r-s)^{2}\right]\right)^{1 / 2}} d x .
\end{aligned}
$$

Define

$$
\omega(r, s ; x)=\frac{4 \chi_{\{|r-s|<|x|<r+s\}}(x)}{\left(\left[(r+s)^{2}-|x|^{2}\right]\left[|x|^{2}-(r-s)^{2}\right]\right)^{1 / 2}}
$$


so that (2) is

$$
\iiint h^{2}(x, \gamma(r)+\gamma(s)) \omega(r, s ; x) d x\left(r \gamma^{\prime}(r) s \gamma^{\prime}(s)\right)^{1 / 2} d r d s
$$

For fixed $y \in(0, \infty)$ the equation $\gamma(r)+\gamma(s)=y$ defines a curve in $\{(r, s): r, s>0\}$. With $x$ fixed as well, write $C_{x, y}$ for the intersection of that curve with $\{|r-s|<$ $|x|<r+s\}$. If $t$ denotes arclength on $C_{x, y}$, then the coarea formula ([3], Theorem 3.2.12) shows that (3) is equal to

$$
\iint h^{2}(x, y) \int_{C_{x, y}} \omega(r, s ; x) \frac{\left(r \gamma^{\prime}(r) s \gamma^{\prime}(s)\right)^{1 / 2}}{\left(\gamma^{\prime}(r)^{2}+\gamma^{\prime}(s)^{2}\right)^{1 / 2}} d t d x d y .
$$

Thus, in order to bound (3) by $C\|h\|_{2}^{2}$ and therefore to establish (1), it is sufficient (and necessary) to prove that

$$
\int_{C_{x, y}} \omega(r, s ; x) \frac{\left(r \gamma^{\prime}(r) s \gamma^{\prime}(s)\right)^{1 / 2}}{\left(\gamma^{\prime}(r)^{2}+\gamma^{\prime}(s)^{2}\right)^{1 / 2}} d t \leq C
$$

for some absolute constant $C$. Also, by symmetry it is enough to replace $C_{x, y}$ by $C_{x, y} \cap\{r>s\}$. So fix $x \in \mathbb{R}^{2}, y \in(0, \infty)$. Recall that $t$ denotes arclength on $C_{x, y}$, and let $(r(t), s(t))$ be a parametrization of $C_{x, y}$ normalized so that $r(0)=s(0)$ and $r^{\prime}(t) \geq 0$. We will eventually need some facts about the derivatives of $r(t)$ and $s(t)$. Since $\gamma(r(t))+\gamma(s(t)) \equiv y$, it follows that $\gamma^{\prime}(r(t)) r^{\prime}(t)+\gamma^{\prime}(s(t)) s^{\prime}(t) \equiv 0$. Since $\gamma^{\prime}>0$ and $r^{\prime}(t)>0$, we conclude that $s^{\prime}(t)<0$. Also, since $r(t)>s(t)$ and $\gamma^{\prime \prime}>0$, it follows that $\left|s^{\prime}(t)\right|>r^{\prime}(t)$. Thus

$$
r^{\prime}(t)+s^{\prime}(t)<0
$$

Differentiating $r^{\prime}(t)^{2}+s^{\prime}(t)^{2} \equiv 1$ shows that $\left[r^{\prime \prime}(t), s^{\prime \prime}(t)\right]$ is parallel to $\left[\gamma^{\prime}(r(t))\right.$, $\left.\gamma^{\prime}(s(t))\right]$, and it follows that

$$
r^{\prime \prime}(t)+s^{\prime \prime}(t)<0 .
$$

Now define

$$
\begin{aligned}
& C_{1}=C_{x, y} \cap\left\{r^{2}+s^{2} \geq|x|^{2}\right\}, \\
& C_{2}=C_{x, y} \cap\left\{r^{2}+s^{2}<|x|^{2}\right\} .
\end{aligned}
$$

We will establish the analogues of (4) for $C_{1}$ and $C_{2}$. If $r^{2}+s^{2} \geq|x|^{2}$, then

$$
(r+s)^{2}-|x|^{2} \geq 2 r s
$$

and so, since $2 \gamma^{\prime}(r) \gamma^{\prime}(s) \leq \gamma^{\prime}(r)^{2}+\gamma^{\prime}(s)^{2}$, the integral

$$
\int_{C_{1}} \omega(r, s ; x) \frac{\left(r \gamma^{\prime}(r) s \gamma^{\prime}(s)\right)^{1 / 2}}{\left(\gamma^{\prime}(r)^{2}+\gamma^{\prime}(s)^{2}\right)^{1 / 2}} d t
$$

is controlled by

$$
C \int_{C_{1}} \frac{1}{\left(|x|^{2}-(r-s)^{2}\right)^{1 / 2}} d t
$$

where $C$ denotes an absolute constant which may, however, increase from line to line. Since $r^{\prime}(t)^{2}+s^{\prime}(t)^{2} \equiv 1$ and $s^{\prime}(t) \leq 0 \leq r^{\prime}(t)$, it follows that

$$
1 \leq r^{\prime}(t)-s^{\prime}(t) \leq 2^{1 / 2} \text {. }
$$


Thus the change of variable $u=r(t)-s(t)$ bounds the last integral by

$$
\int_{0}^{|x|} \frac{1}{\left(|x|^{2}-u^{2}\right)^{1 / 2}} d u=\frac{\pi}{2}
$$

To treat the integral over $C_{2}$ we begin similarly by noting that if $r^{2}+s^{2}<|x|^{2}$, then $|x|^{2}-(r-s)^{2} \geq 2 r s$. It follows that

$$
\int_{C_{2}} \omega(r, s ; x) \frac{\left(r \gamma^{\prime}(r) s \gamma^{\prime}(s)\right)^{1 / 2}}{\left(\gamma^{\prime}(r)^{2}+\gamma^{\prime}(s)^{2}\right)^{1 / 2}} d t
$$

is bounded by

$$
\begin{array}{r}
\int_{0}^{t_{0}} \frac{(r(t) s(t))^{1 / 2}}{(r(t)+s(t))^{1 / 2} \mid\left(r(t)+s(t)-r\left(t_{0}\right)-\left.s\left(t_{0}\right)\right|^{1 / 2}\right.}\left(\frac{\gamma^{\prime}(r(t)) \gamma^{\prime}(s(t))}{r(t) s(t)}\right)^{1 / 2} \\
\times \frac{d t}{\left(\gamma^{\prime}(r(t))^{2}+\gamma^{\prime}(s(t))^{2}\right)^{1 / 2}}
\end{array}
$$

for some $t_{0}$. It follows from (5) and (6) that $\left|r^{\prime}(t)+s^{\prime}(t)\right|$ is an increasing function of $t$ so that, if $0<t<t_{0}$, we have

$$
\left|r(t)+s(t)-r\left(t_{0}\right)-s\left(t_{0}\right)\right| \geq\left|t_{0}-t\right|\left|r^{\prime}(t)+s^{\prime}(t)\right| .
$$

The equations $\gamma^{\prime}(r(t)) r^{\prime}(t)+\gamma^{\prime}(s(t)) s^{\prime}(t) \equiv 0$ and $r^{\prime}(t)^{2}+s^{\prime}(t)^{2} \equiv 1$ show that

$$
\left|r^{\prime}(t)+s^{\prime}(t)\right|=\frac{\gamma^{\prime}(r(t))-\gamma^{\prime}(s(t))}{\left[\gamma^{\prime}(r(t))^{2}+\gamma^{\prime}(s(t))^{2}\right]^{1 / 2}} .
$$

The normalization $r(0)=s(0)$ and (7) together imply that $|r(t)-s(t)| \geq t$. With (9) and (10), this gives

$$
\left|r(t)+s(t)-r\left(t_{0}\right)-s\left(t_{0}\right)\right| \geq \frac{\gamma^{\prime}(r(t))-\gamma^{\prime}(s(t))}{|r(t)-s(t)|} \frac{\left|t_{0}-t\right| t}{\left[\gamma^{\prime}(r(t))^{2}+\gamma^{\prime}(s(t))^{2}\right]^{1 / 2}} .
$$

Writing $r$ and $s$ for $r(t)$ and $s(t)$, the integrand in (8) is thus bounded by

$$
\begin{aligned}
\frac{(r s)^{1 / 2}}{(r+s)^{1 / 2}}\left(\frac{(r-s)\left(\gamma^{\prime}(r)^{2}+\gamma^{\prime}(s)^{2}\right)^{1 / 2}}{t\left(t_{0}-t\right)\left(\gamma^{\prime}(r)-\gamma^{\prime}(s)\right)}\right)^{1 / 2}\left(\frac{\gamma^{\prime}(r) \gamma^{\prime}(s)}{r s}\right)^{1 / 2} \frac{1}{\left(\gamma^{\prime}(r)^{2}+\gamma^{\prime}(s)^{2}\right)^{1 / 2}} \\
=\frac{(r s)^{1 / 2}}{(r+s)^{1 / 2}}\left(\frac{\gamma^{\prime}(r) \gamma^{\prime}(s)}{r s}\right)^{1 / 4} \frac{1}{\left(\gamma^{\prime}(r)^{2}+\gamma^{\prime}(s)^{2}\right)^{1 / 4}} \frac{1}{\left(t\left(t-t_{0}\right)\right)^{1 / 2}} \\
\times\left(\frac{(r-s)}{\left(\gamma^{\prime}(r)-\gamma^{\prime}(s)\right.}\right)^{1 / 2}\left(\frac{\gamma^{\prime}(r) \gamma^{\prime}(s)}{r s}\right)^{1 / 4} .
\end{aligned}
$$

Now, since $\gamma^{\prime \prime}$ is increasing,

$$
\left(\frac{1}{r} \int_{0}^{r} \gamma^{\prime \prime}(u) d u \cdot \frac{1}{s} \int_{0}^{s} \gamma^{\prime \prime}(u) d u\right)^{1 / 2} \leq \frac{1}{r-s} \int_{s}^{r} \gamma^{\prime \prime}(u) d u .
$$

It follows that

$$
(r-s)\left(\gamma^{\prime}(r) \gamma^{\prime}(s)\right)^{1 / 2} \leq(r s)^{1 / 2}\left(\gamma^{\prime}(r)-\gamma^{\prime}(s)\right) .
$$


Therefore (8) is bounded by $C$ times

$$
\int_{0}^{t_{0}} \frac{1}{\left(t\left(t_{0}-t\right)\right)^{1 / 2}} d t=\pi .
$$

\section{REFERENCES}

[1] J.-G. Bak, Restrictions of Fourier transforms to flat curves in $\mathbb{R}^{2}$, Illinois J. of Math. 38 (1994), 327-346. MR 94m:42032

[2] A. Carbery and S. Ziesler, Restriction and decay for flat hypersurfaces, Publ. Mat. 46 (2002), 405-434. MR 2003i:42019

[3] H. Federer, Geometric Measure Theory, Die Grundlehren der mathematischen Wissenschaften, Band 153, Springer-Verlag, New York, 1969. MR 41:1976

[4] A. Greenleaf, Principal curvature and harmonic analysis, Indiana Univ. Math. J. 30 (1981), 519-537. MR 84i:42030

[5] E. M. Stein, Harmonic Analysis: real-variable methods, orthogonality, and oscillatory integrals, Princeton University Press, Princeton, NJ, 1993. MR 95c:42002

Department of Mathematics, Florida State University, Tallahassee, Florida 32306 4510

E-mail address: oberlin@math.fsu.edu 